\title{
(6) OPEN ACCESS \\ Drug use and upper gastrointestinal complications in children: a case-control study
}

${ }^{1}$ Regina Margherita Children's Hospital, Turin, Italy ${ }^{2}$ Anna Meyer Children's University Hospital, Florence, Italy

${ }^{3}$ Santobono Paediatric Hospital, Naples, Italy ${ }^{4}$ National Centre for Epidemiology, Italian National Institute of Health, Rome, Italy

\section{Correspondence to}

Dr Francesca Menniti-Ippolito, National Centre for

Epidemiology, National Institute of Health, Viale Regina Elena 299, Rome 00161, Italy;

francesca.menniti@iss.it

Received 26 March 2012 Revised 25 October 2012 Accepted 26 October 2012 Published Online First 21 December 2012

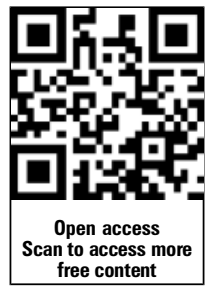

To cite: Bianciotto $\mathrm{M}$ Chiappini E, Raffaldi I, et al. Arch Dis Child

2013;98:218-221

\author{
Manuela Bianciotto, ${ }^{1}$ Elena Chiappini, ${ }^{2}$ Irene Raffaldi, ${ }^{1}$ Clara Gabiano, ${ }^{1}$ \\ Pier-Angelo Tovo, ${ }^{1}$ Sara Sollai, ${ }^{2}$ Maurizio de Martino, ${ }^{2}$ Francesco Mannelli, ${ }^{2}$ \\ Vincenzo Tipo, ${ }^{3}$ Roberto Da Cas, ${ }^{4}$ Giuseppe Traversa, ${ }^{4}$ Francesca Menniti-Ippolito, ${ }^{4}$ \\ and the Italian Multicenter Study Group for Drug and Vaccine Safety in Children
}

\section{ABSTRACT}

Objective To evaluate the risk of upper gastrointestinal complications (UGIC) associated with drug use in the paediatric population.

Methods This study is part of a large Italian prospective multicentre study. The study population included children hospitalised for acute conditions through the emergency departments of eight clinical centres. Patients admitted for UGIC (defined as endoscopically confirmed gastroduodenal lesions or clinically defined haematemesis or melena) comprised the case series; children hospitalised for neurological disorders formed the control group. Information on drug and vaccine exposure was collected through parental interview during the children's hospitalisation. Logistic regression was used to estimate ORs for the occurrence of UGIC associated with drug use adjusted for age, clinical centre and concomitant use of any drug. Results 486 children hospitalised for UGIC and 1930 for neurological disorders were enrolled between November 1999 and November 2010. Drug use was higher in cases than in controls ( $73 \%$ vs $54 \%$; $p<0.001)$. UGICs were associated with the use of nonsteroidal anti-inflammatory drugs (NSAIDs) (adjusted OR $2.9,95 \% \mathrm{Cl} 2.1$ to 4.0 ), oral steroids (adjusted OR 2.9, $95 \% \mathrm{Cl} 1.7$ to 4.8 ) and antibiotics (adjusted OR 2.3, $95 \% \mathrm{Cl} 1.8$ to 3.1). The duration of use of these drug categories was short (range 1-8 days). Paracetamol showed a lower risk (adjusted OR 2.0, 95\% Cl 1.5 to 2.6) compared to ibuprofen (adjusted OR $3.7,95 \% \mathrm{Cl}$ 2.3 to 5.9), although with partially overlapping Cls. Conclusions NSAIDs, oral steroids and antibiotics, even when administered for a short period, were associated with an increased risk of UGIC.

\section{INTRODUCTION}

Mucosal damage to the gastrointestinal tract may lead to a wide spectrum of disorders, from asymptomatic minimal lesions, to severe, potentially fatal, complications. ${ }^{1}$ Upper gastrointestinal complications (UGICs) such as bleeding are often associated with drug use, especially non-steroidal antiinflammatory drugs (NSAIDs), oral steroids and oral anticoagulants. ${ }^{2} 3$

NSAIDs inhibit the cyclooxygenase enzymes catalysing the production of prostaglandins and thromboxane, with consequent reduced release of gastric mucus, inhibition of bicarbonate production and alteration of local microcirculation. ${ }^{4}$ Steroids can also enhance gastrin secretion and induce

\section{What is already known on this topic}

- Gastrointestinal toxicity in association with non-steroidal anti-inflammatory drugs (NSAIDs) and corticosteroids in adults is well documented.

- There are only a few studies describing the association between gastrointestinal toxicity and NSAID use in children.

\section{What this study adds}

- Our study confirms a threefold increased risk of upper gastrointestinal complications (UGIC) associated with either NSAID or oral corticosteroid use.

- A twofold increased risk of UGIC was estimated for paracetamol and antibiotics.

parietal cell hyperplasia with increased acid production. ${ }^{5}$

In adults, a small number of studies have specifically examined the association between corticosteroids and gastrointestinal toxicity ${ }^{6-8}$ and there is an extensive body of literature on gastrointestinal complications related to drug consumption, but few studies have focused on children. ${ }^{5-13}$

Since 1999 active surveillance of the role of drugs and vaccines in the occurrence of specific clinical conditions responsible for the hospitalisation of paediatric patients has been conducted in Italy. This surveillance is useful for highlighting or confirming adverse effects associated with drug and vaccine use in children. ${ }^{14-17}$

In the present article we focus on the occurrence of hospitalisation for UGIC in association with drugs frequently used in the paediatric population, with particular emphasis on antipyretics/analgesics, steroids and antibiotics.

\section{METHODS}

\section{Study population}

The study population consists of all children admitted through the emergency departments (ED) of eight Italian paediatric hospitals in Genoa, Turin, Padua, Florence, Rome (two hospitals), Naples and 
Palermo between 1 November 1999 and 30 November 2010. Children were enrolled regardless of drug and vaccine use, if they had the following conditions: endoscopically confirmed UGIC (or clinical haematemesis and melena); neurological disorders; non-infectious muco-cutaneous diseases and vasculitis; or thrombocytopenia (platelet count $<100000 / \mu \mathrm{l}$ ). Children with concomitant diagnoses of cancer or immunodeficiency and age less than 1 month were excluded.

Information on the reason for hospital admission was derived from the ED records, and the final diagnosis was retrieved from the clinical records after discharge. UGIC cases comprise all children whose reason for admission included haematemesis/ melena and children who had received an endoscopy during hospitalisation and were discharged with a diagnosis of gastroduodenal lesion. The following conditions were defined as gastroduodenal lesions on endoscopy: gastric and/or duodenal ulcer, and gastric and/or duodenal erosions (with or without bleeding).

Parents were interviewed, using a structured questionnaire, during the hospital admission of their child. All medicines administered in the previous 3 weeks and all vaccines received in the previous 12 weeks were noted. Information on drug use, including indication, dose, duration of treatment, prescriber (physician or self-medication) and other personal data (age, gender, weight, height, chronic diseases, parents' education) was also collected.

\section{Data analysis}

ORs of clinically defined (haematemesis and melena) or endoscopically confirmed UGIC associated with drug use were estimated according to a case-control study design. Drug exposure to specific drugs or drug categories in children admitted with UGIC (cases) was compared to exposure in children admitted for neurological disorders (controls). This control group was chosen because drug exposure in children hospitalised for neurological disorders accurately reflects drug exposure in the population from which UGIC cases were derived.

Pre-existing chronic diseases were divided into six categories: allergies, gastrointestinal diseases (gastroesophageal reflux, coeliac disease, ulcerative colitis), haematological disorders, recurrent infections, neurological problems and birth defects. Indications for drug use were classified into nine groups: allergy, asthma, pain or headache, fever, gastrointestinal diseases, otitis, upper respiratory tract infections (URTI), prophylaxis and other indications. The use of oral steroids was categorised as appropriate or not, according to the approved indications; we considered as appropriate prescriptions for asthma and allergy, while all prescriptions for other indications (eg, fever, cough, otitis, pharyngitis) were classified as inappropriate.

Categorical variables, presented as numbers and percentages, were compared using the $\chi^{2}$ test, while continuous variables, reported as median (range), were evaluated using the MannWhitney $\mathrm{U}$ test. All tests were two sided and significance was set at $\mathrm{p}<0.05$.

To estimate the adjusted ORs and related 95\% CIs, we used the multivariate logistic regression model. ORs were adjusted for age, clinical centre, calendar year, presence of chronic diseases and concomitant use of any other drug. Year of inclusion in the study and chronic diseases did not modify the estimates and, thus, were not included in the final logistic model. Statistical analysis was performed using SPSS V.17.0 (SPSS, Chicago, Illinois, USA).

\section{Organisation and ethics}

The protocol of the study was approved by the ethics committee of each clinical centre. Informed consent for the use of data for research purposes was obtained before parents were interviewed. The study was coordinated by the National Centre of Epidemiology of the National Institute of Health.

\section{RESULTS}

A total of 486 children hospitalised for UGIC and 1930 for neurological disorders were enrolled in the study. Of the children hospitalised for UGIC, 324 (66.6\%) were admitted for haematemesis, 31 (6.4\%) for melena, and 29 (6.0\%) for both haematemesis and melena. The remaining 102 children (21.0\%) who were admitted for symptoms compatible with gastroduodenal lesions (other than haematemesis/melena), such as abdominal pain and vomiting, underwent endoscopy during hospitalisation and were discharged with a diagnosis of UGIC. An endoscopy was performed in 248 (51\%) of the 486 UGIC cases.

The most frequent diagnoses in the 1930 children included in the control group were afebrile convulsions (822, 43\%), disturbances of consciousness (such as syncope, faintness, dizziness, somnolence) $(530,27 \%)$, peripheral neuropathy $(78,4 \%)$ and extrapyramidal symptoms $(65,3 \%)$.

The majority of cases (302 children, 62.1\%) were admitted to Santobono Hospital (Naples). The main characteristics of cases and controls are summarised in table 1 . Males were equally represented in both groups, and cases were 5 months younger than controls $(p=0.01)$. However, age did not have a confounding effect when taken into account in the logistic model. Seventy-five of the 486 cases (15.4\%) had concomitant chronic diseases versus 265 of the 1930 controls $(13.7 \%)(p=0.37)$.

Chronic gastrointestinal diseases were uncommon, although more frequent in cases than in controls $(2.5 \%$ vs $1.1 \%$; $\mathrm{p}=0.06$ ). The presence of such conditions was an independent

\begin{tabular}{|c|c|c|c|c|c|}
\hline & $\begin{array}{l}\text { Cases } \\
(\mathrm{N}=486)\end{array}$ & $\%$ & $\begin{array}{l}\text { Controls } \\
(\mathrm{N}=1930)\end{array}$ & $\%$ & $p$ Value \\
\hline $\begin{array}{l}\text { Median age, months } \\
\text { (25th-75th percentile) }\end{array}$ & $41(15-71)$ & & $46(11-115)$ & & 0.01 \\
\hline Male & 274 & 56.4 & 1002 & 51.9 & 0.08 \\
\hline $\begin{array}{l}\text { Median LoS, days } \\
\text { (25th-75th percentile) }\end{array}$ & $3(2-5)$ & & $3(2-6)$ & & 0.69 \\
\hline Chronic diseases & 75 & 15.4 & 265 & 13.7 & 0.37 \\
\hline Allergy & 47 & 9.7 & 168 & 8.7 & 0.98 \\
\hline Gastrointestinal disease & 12 & 2.5 & 21 & 1.1 & 0.06 \\
\hline Haematological disorders & 4 & 0.8 & 7 & 0.4 & 0.42 \\
\hline Recurrent infections & 3 & 0.6 & 12 & 0.6 & 0.90 \\
\hline Neurological problems & 8 & 1.6 & 40 & 2.1 & 0.43 \\
\hline Birth defects & 10 & 2.1 & 41 & 2.1 & 0.78 \\
\hline $\begin{array}{l}\text { Drug exposure } \\
\text { (at least one) }\end{array}$ & 353 & 72.6 & 1042 & 54.0 & $<0.001$ \\
\hline \multicolumn{6}{|l|}{ Indication for use of drugs* } \\
\hline URTI & 141 & 39.9 & 259 & 24.9 & $<0.001$ \\
\hline Fever & 56 & 15.9 & 97 & 9.3 & $<0.001$ \\
\hline Asthma & 13 & 3.7 & 15 & 1.4 & 0.001 \\
\hline Allergy & - & - & 19 & 1.8 & \\
\hline
\end{tabular}


risk factor for UGIC manifestations with an adjusted OR of 2.3 (95\% CI 1.1 to 4.9 ). However, after adjustment for the personal history of pre-existing gastrointestinal diseases, no confounding effect was found on the estimates. The number of children who had received at least one drug within the 3 weeks preceding the hospital admission was 353 among cases and 1042 among controls $(73 \%$ vs $54 \% ; \mathrm{p}<0.001)$. The mean number of drugs per child was 1.7 among cases and 1.1 among controls $(\mathrm{p}<0.001)$. Approximately $17 \%$ of these drugs were consumed without medical advice in both groups. The drugs reported in the personal history were those most frequently prescribed in the paediatric population in Italy, that is, antibiotics, paracetamol, NSAIDs and steroids.

Duration of use was quite short and similar for all study drugs. The median duration for NSAID administration was 3 days in cases and 2 days in controls $(p=0.41)$. One child (4 years old) admitted with haematemesis had been treated for 80 days with indomethacin and ibuprofen for arthritis. The median duration of use for steroids was 3 days in cases and 4 days in controls $(p=0.16)$. The indication was reported in 79 of the 87 steroid users (91\%); in 17 children (21.5\%) it was an approved indication (eg, asthma, urticaria).

An increased risk of UGIC was found for the use of NSAIDs, paracetamol, corticosteroids and antibiotics. The adjusted OR among NSAID users was 2.9 (95\% CI 2.1 to 4.0). The risk estimates of individual NSAIDs and paracetamol presented largely overlapping CIs (table 2).

On the basis of the number of children who visited the ED of the participating centres in the year 2010, we calculate that around 2.1 million children were treated in the participating EDs during the study period. Thus, we can roughly estimate

Table 2 ORs and Cls of upper gastrointestinal complications in association with drugs*

\begin{tabular}{lccl}
\hline Drugs & $\begin{array}{l}\text { Cases } \\
\text { (N=486) } \\
\mathbf{n}\end{array}$ & $\begin{array}{l}\text { Controls } \\
\text { (N=1930) } \\
\mathbf{n}\end{array}$ & $\begin{array}{l}\text { Adjusted ORt } \\
\text { (95\% Cl) }\end{array}$ \\
\hline Antibiotics & 135 & 242 & $2.3(1.8$ to 3.1$)$ \\
Amoxicillin+clavulanic & 44 & 77 & $2.3(1.5$ to 3.5$)$ \\
acid & & & $2.0(1.0$ to 3.8$)$ \\
Claritromicin & 17 & 30 & $1.8(0.9$ to 3.4$)$ \\
Amoxicillin & 16 & 30 & $1.7(0.8$ to 3.5$)$ \\
Cefaclor & 14 & 24 & $1.9(1.4$ to 2.7$)$ \\
Steroids & 87 & 140 & $2.9(1.7$ to 4.8$)$ \\
Oral steroids & 39 & 30 & $1.0(0.6$ to 1.7$)$ \\
Inhaled steroids & 24 & 81 & $3.0(1.5$ to 5.9$)$ \\
Oral+inhaled steroids & 20 & 18 & $2.9(2.1$ to 4.0$)$ \\
NSAIDs & 81 & 121 & $3.7(2.3$ to 5.9$)$ \\
Ibuprofen & 40 & 51 & $1.6(0.8$ to 3.2$)$ \\
Niflumic acid & 14 & 23 & $2.6(1.2$ to 5.6$)$ \\
Ketoprofen & 12 & 23 & $2.0(1.5$ to 2.6$)$ \\
Paracetamol & 119 & 294 & $3.1(1.5$ to 6.2$)$ \\
Paracetamol & 16 & 21 & $2.5(0.9$ to 7.4$)$ \\
+ibuprofen & & & $0.8(0.7$ to 1.1$)$ \\
Aspirin & 6 & 12 & \\
Other drugs & 186 & 746 & \\
Total drug use & 353 & 1042 & \\
\hline *Al drats & & & \\
\hline
\end{tabular}

${ }^{*}$ All drugs with at least 10 exposed in the control group.

†Adjusted for age, clinical centre and concomitant use of other drugs.

NSAIDs, non-steroidal anti-inflammatory drugs; UGIC, upper gastrointestinal

complications. that during the study period around 2.4 per 10000 children with ED visits were admitted for UGIC.

\section{DISCUSSION}

In this article we provided evidence of an increased risk of UGICs associated with drug use in the paediatric population.

The proportion of children who had received at least one drug in the 3 weeks preceding hospital admission was higher among cases than among controls. Significantly increased risks were estimated for NSAIDs, steroids and antibiotics. In agreement with our findings, an increased risk for NSAIDs was also observed in a case-crossover study of 177 children with gastrointestinal bleeding. ${ }^{9}$ The adjusted OR for NSAIDs reached 8.2 (95\% CI 2.6 to 26.0), with one third of cases attributable to exposure to NSAIDs administered at analgesic or antipyretic doses. ${ }^{9}$ However, as underlined by the authors, the study has several limitations and the estimated risks appear higher than those observed in adults, ${ }^{2}$ since the findings may have been influenced by the limited dataset and a recall bias could not be excluded. ${ }^{9}$

Among NSAIDs, ibuprofen was the most commonly used drug. Although ibuprofen is safe in most children, ${ }^{18}$ the association between gastrointestinal bleeding and ibuprofen use is well known in both adults $^{2}$ and children. ${ }^{10}$ We estimated an increased risk of UGIC associated with ibuprofen (adjusted OR $3.7,95 \%$ CI 2.3 to 5.9 ). It has to be noted that our estimate is included in the large CI of the case-crossover study ${ }^{9}$ and overlaps with findings reported in adults (a twofold to fourfold increase in relative risks). ${ }^{2}$ It is assumed that gastrointestinal bleeding is associated with chronic use of NSAIDs. ${ }^{19}$ However, in our study the mean duration of ibuprofen use in cases was 4 days, so UGIC may occur even during a short course therapy. We confirm the lower level of gastrotoxicity, in comparison with NSAIDs, of paracetamol, the most widely used drug in the paediatric population in Italy.

Steroid prescribing was off-label in a large proportion of cases and was mostly used for URTI or fever. This finding underlines the need for educational programmes in Italy to promote the correct use of steroids in children. Notably, the association with UGIC was limited to oral steroid use as no increased risk was observed among users of inhaled steroids.

Although poorly documented in the literature, gastrointestinal adverse reactions, featuring diarrhoea, nausea and abdominal pain, related to antibiotic administration, are usually mild and reversible with treatment cessation. ${ }^{20} 21$ There are very few data on the upper gastrointestinal toxicity of antibiotics. Immune -mediated thrombocytopenia is often implicated in bleeding mechanisms associated with antibiotics, in particular $\beta$-lactams. ${ }^{22}$ In our study, however, children hospitalised for UGIC did not have a concomitant diagnosis of thrombocytopenia. The reason for an increased risk of UGIC among antibiotic users, after taking into account the effect of age and concomitant use of other drugs, remains unclear. ${ }^{11} 2223$

Our study has several potential limitations. Cases were significantly younger than controls; however, the risk estimates did not change after age was taken into account in the logistic model. An endoscopy was performed only in half of the children diagnosed with UGIC; however, all cases without endoscopic confirmation had haematemesis or melena at admission. The risk estimates did not change when the analysis was restricted to children who underwent endoscopy.

As in other case-control studies, the possibility of a recall bias cannot be excluded. However, such a bias cannot explain the observed risk estimates since the ascertainment of exposure was based on the same procedure in cases and controls (interviewing 
parents during their child's hospitalisation). Children hospitalised for neurological problems are a suitable control group for patients with UGICs. Both cases and controls were admitted through EDs for an acute condition and, consequently, drug use preceding symptoms causing hospitalisation should not differ between cases and controls.

Pre-existing chronic conditions were unlikely to have influenced the results, since the proportions of children with chronic conditions were similar in the two groups. However, the small sample size of some subgroups may have limited our ability to draw definitive conclusions regarding some specific drugs.

Our study indicates that UGIC is a rare event (486 children hospitalised in just over 11 years). Although, given the casecontrol design, we could not calculate incidence, the number of UGIC patients admitted through EDs can be roughly estimated as 2.4 per 10000 children with ED visits. Published incidence data are limited to the paediatric intensive care unit population, ${ }^{24}$ which cannot be compared with children who develop an UGIC as outpatients. Our study suggests that the absolute risk of UGIC is low, nevertheless it remains to be determined if all prescriptions were really necessary. The appropriate use of drugs in children should be promoted in order to prevent the occurrence of serious adverse events.

\section{The Italian Multicenter Study Group for Drug and Vaccine Safety in}

Children The Italian Multicenter Study Group for Drug and Vaccine Safety in Children: Francesca Menniti-Ippolito, Roberto Da Cas, Luciano Sagliocca, Giuseppe Traversa (National Center for Epidemiology, National Institute of Health, Rome); Fernanda Ferrazin, Carmela Santuccio, Loriana Tartaglia, Francesco Trotta (Italian Medicines Agency, Rome); Pasquale Di Pietro, Salvatore Renna, Rossella Rossi, Bianca Domenichini, Stefania Gamba, Francesco Trovato (Giannina Gaslini Paediatric Hospital, Genoa); Pier-Angelo Tovo, Manuela Bianciotto, Carmelina Calitri, Clara Gabiano, Irene Raffaldi, Antonio Urbino (Regina Margherita Paediatric Hospital, Turin); Liviana Da Dalt, Valentina Favero, Laura Giordano, Maura Baraldi, Federica Bertuola, Eleonora Lorenzon, Francesca Parata, Giorgio Perilongo, Silvia Vendramin (Department of Paediatrics, University of Padua); Monica Frassineti, Anna Maria Calvani, Elena Chiappini, Maurizio De Martino, Claudia Fancelli, Francesco Mannelli, Rachele Mazzantini, Sara Sollai, Elisabetta Venturini (Anna Meyer Children's University Hospital, Florence); Nicola Pirozzi, Umberto Raucci, Antonino Reale, Rossella Rossi (Emergency Department, Bambino Gesù Children's Hospital, Rome); Nadia Mores, Giulia Bersani, Alessia De Nisco, Antonio Chiaretti, Riccardo Riccardi, Costantino Romagnoli (Pharmacology and Paediatrics and Developmental Neuroscience, Università Cattolica S. Cuore, Rome); Vincenzo Tipo, Michele Dinardo, Teresa Pisapia (Santobono Paediatric Hospital, Naples); Annalisa Capuano, Elisabetta Parretta, Concita Rafaniello (Department of Experimental Medicine, Section of Pharmacology 'L. Donatelli', Second University of Naples, Naples); Fortunata Fucà, Eleonora Di Rosa (Giovanni Di Cristina Paediatric Hospital, Palermo).

Funding This study was funded by the Italian Medicines Agency (AIFA) (Fasc. ISS-T7H).

\section{Competing interests None.}

Provenance and peer review Not commissioned; externally peer reviewed.

Open Access This is an Open Access article distributed in accordance with the Creative Commons Attribution Non Commercial (CC BY-NC 3.0) license, which permits others to distribute, remix, adapt, build upon this work non-commercially, and license their derivative works on different terms, provided the original work is properly cited and the use is non-commercial. See: http://creativecommons.org/ licenses/by-nc/3.0/

\section{REFERENCES}

1 Carvalho ED, Nita MH, Paiva LM, et al. Gastrointestinal bleeding. J Pediatr 2000;1: S135-46.

2 Garcia Rodriguez LA, Hernandez-Diaz S. The risk of upper gastrointestinal complications associated with nonsteroidal anti-inflammatory drugs, glucocorticoids, acetaminophen, and combination of these agents. Arthritis Res 2001;3:98-101.

3 Pirmohamed $\mathrm{M}$, James $\mathrm{S}$, Meakin $\mathrm{S}$, et al. Adverse drug reaction as cause of admission to hospital: prospective analysis of 18820 patients. BMJ 2004:329:15-19.

4 Al Mofleh IA, Al Rashed RS. Nonsteroidal antinflammatory drug-induced gastrointestinal injuries and related adverse reactions: epidemiology, pathogenesis and management. Saudi J Gastroenterol 2007;13:107-13.

5 Luo JC, Chang FY, Chen TS, et al. Gastric mucosal injury in systemic lupus erithematosus patients receiving methylprednisolone therapy. Br J Clin Pharmacol 2009;68:252-9.

6 Piper JM, Ray WA, Daugherty JR, et al. Corticosteroid use and peptic ulcer disease: role of nonsteroidal anti-inflammatory drugs. Ann Intern Med 1991;114:735-40.

7 Hernández-Díaz S, Rodríguez LA. Steroids and risk of upper gastrointestinal complications. Am J Epidemiol 2001;153:1089-93.

8 Messer J, Reitman D, Sacks HS, et al. Association of adrenocorticosteroid therapy and peptic-ulcer disease. NEJM 1983;309:21-4.

9 Grimaldi-Bensouda L, Abenhaim L, Michaud L, et al. Clinical features and risk factors for upper gastrointestinal bleeding in children: a case-crossover study. Eur J Clin Pharmacol 2010;66:831-7.

10 Autret-Leca E, Bensouda-Grimaldi L, Maurage C, et al. Upper gastrointestinal complications associated with NSAIDs in children. Therapie 2007;62:173-6.

11 Ozen IO, Moralioglu S, Karabulut R, et al. Cefoperazone induced gastro-intestinal haemorrhage. A case report. Acta Chir Belg 2008;108:777-8.

12 Principi N, Esposito S. Comparative tolerability of erythromycin and newer macrolide antibacterials in paediatric patients. Drug Saf 1999;20:25-41.

13 Klein JO. History of macrolide use in pediatrics. Pediatr Infect Dis $J$ 1997;16:427-31

14 Menniti-Ippolito F, Sagliocca L, Da Cas R, et al. Niflumic acid and cutaneous reactions in children. Arch Dis Child 2001:84:430-1.

15 Menniti-Ippolito F, Traversa G, Da Cas R, et al. Extrapyramidal reactions in children treated with metoclopramide. Ital J Pediatr 2004:30:49-52.

16 Bertuola F, Morando C, Menniti-Ippolito F, et al. Association between drug and vaccine use and acute immune thrombocytopenia in childhood. A case control study in Italy. Drug Saf 2010;33:65-72.

17 Menniti-Ippolito F, Da Cas R, Sagliocca L, et al. Effectiveness and safety of the A-H1N1 vaccine in children: a hospital-based case-control study. BMJ Open 2011;2: e000167.

18 Southey ER, Soares-Weiser K, Kleijnen J, et al. Systematic review and meta-analysis of the clinical safety and tolerability of ibuprofen compared with paracetamol in paediatric pain and fever. Curr Med Res Opin 2009;25:2207-2

19 Berezin SH, Bostwick HE, Halata MS, et al. Gastrointestinal bleeding in children following ingestion of low dose ibuprofen. J Pediatr Gastroenterol Nutr 2007;44:506-8

20 Rouveix B. Antibiotic safety assessment. Int J Antimicrob Agents 2003;21:215-21.

21 Lode $\mathrm{H}$. Safety and tolerability of commonly prescribed oral antibiotics for the treatment of respiratory tract infections. Am J Med 2010;123:S26-38.

22 Shevchuk YM, Conly JM. Antibiotics-associated hypoprothrombinemia: a review of prospective studies, 1966-1988. Rev Infect Dis 1990;12:1109-26.

23 Schetag JJ, Welage LS, Grasela TH, et al. Determinants of antibiotic-associated hypoprothrombinemia. Pharmacotherapy 1987;7:80-6.

24 Victor L, Fox MD. Gastrointestinal bleeding in infancy and childhood. Gastroenterol Clin North Am 2000;29:37-66. 